\title{
MORPHOLOGICAL DIVERSIFICATION OF LATE DEVONIAN AND CARBONIFEROUS SEEDS
}

SIMS, Hallie J.* University of Chicago, Department of the Geophysical Sciences, 5734 S. Ellis Ave, Chicago, IL 60637, U.S.A.

Innovations in the reproductive system have been used to explain nearly every floral radiation in the Phanerozoic. However, although questions examining the relation between propagule dispersal and diversification have received considerable attention in modern ecological research, relatively few rigorous analyses have addressed this question in the broader historical context of the fossil record. The innovation of the seed in the late Devonian is thought to have allowed subsequent lineages to expand geographically and ecologically into areas previously uninhabitable because of reduced dependence on water for gamete transfer. Changes in seed size have been thought to represent a compromise between two fairly discrete selective pressures: one to increase the likelihood of reaching favorable establishment conditions, and one to lower the risk of mortality upon arrival at a particular site. Seed volume generally reflects the amount of nutritive reserve contained for the developing embryo and also enhances or constrains dispersal. An analysis of fossil seeds through time provides an opportunity to contrast trends in taxonomic diversity and overall morphological diversity in a discrete organ, with the evolution of specific ecological adaptations that may be related to plant life history.

Morphology of over 100 fossil seed "species" was quantified using a set of discrete characters (binary and multistate, all unordered) to define all features of the seed which were preserved consistently. Morphological disparity was calculated as the mean pairwise distance between species, and the range of morphospace occupied, for eight time intervals spanning the Late Devonian and Carboniferous. Results indicate that morphological disparity increased rapidly during the Late Devonian and early Carboniferous to a 50-My plateau, followed by a decline in disparity in the latest Carboniferous. Similarly, the extremes of occupied morphospace were reached early, with later species filling in and then clustering. In contrast, taxonomic diversity (based on seed taxa only) continued to increase dramatically through the latest Carboniferous, reflecting the radiation of seed plants into diverse ecological situations. Analysis of estimates of seed volume for the same data set indicates that the mean, range and variance also increase dramatically by the latest Carboniferous. The data support the hypothesis of constraints on overall morphological disparity reached early in clade history (even as taxonomic diversity continues to increase), yet do not definitively imply specific mechanisms of constraint. 